\title{
Research on the Layout Optimization of Park and Ride
}

Based on Transport Demand

\author{
Li Yuhong \\ School of Traffic and Transportation \\ Beijing Jiaotong University \\ Beijing, China \\ 12120881@bjtu.edu.cn
}

\author{
He Shiwei \\ School of Traffic and Transportation \\ Beijing Jiaotong University \\ Beijing, China \\ shwhe@bjtu.edu.cn
}

\begin{abstract}
With the problem of urban traffic congestion becoming more worse, we are paying more attention on the Park and Ride(P\&R), which is one aspect of the Transport Demand Management(TDM). As the study on the layout of $P \& R$ is an important part of $P \& R$ planning, it plays a major role in strengthening TDM. On the basis of the traffic network system, this paper designs a layout optimization model, taking the total cost of $P \& R$ construction and person's travel as the minimum optimized goal. In the end, this paper also designs a case about it with solving the model by Lingo mathematics software so as to check the effectiveness of the model.
\end{abstract}

Index Terms-Transport Demand Management; Park and Ride; Layout Optimization; Cost-effectiveness

\section{INTRODUCTION}

With the continuous increases of vehicle numbers, the problem of city traffic congestion becomes more and more serious in recent years. As one of the most important measures of Transport Demand Management, Park and Ride plays a significant role in the relief of urban traffic pressure and the adjustment of urban traffic structure. Research on the layout optimization of Park and Ride is an important part of P\&R program planning, and the rationality of its location is directly related to whether these Traffic Demand Management measures can work economically and efficiently or not. Paper[1] conducts a survey and an analysis of Beijing Park and Ride demand which draws a conclusion that the main parkers are commuters. Paper[2] analyzes the existing problems of Beijing P\&R sites, and points out a phenomena that the abilities of some transfer sites are saturated while some are vacant, then comes to the conclusion that irrational phenomenon exist in its current layout.

In the research on the layout optimization of Park and Ride, Paper[3] proposes an intercepting layout optimization problem in the case that the number of facilities is given and the route and traffic volume of passenger flow are known; Paper[4] applies PIM (Preventative Inspection Model) which is the idea of dangerous goods stations layout to Park and Ride layout optimization; Paper[5] puts forward an economic optimization method based on the target of getting the largest profits and the lowest social costs while considering the layout of Park and Ride in a linear single-center city; Paper[6] presents a layout optimization model of Park and Ride whose objective is to get the minimum total generalized cost between the demand points and the nearest supply points based on a multi-facility and multi-objective model of the Park and Ride location; Paper[7] proposes a location choice model, using the minimum generalized travel cost as the location target, and the model is solved by Genetic Algorithm; Paper[8] introduces an optimum planning theory of the Park and Ride facilities based on a modified flow interception model, which is established through space analysis according to the specialities and functions of P \& R system; Paper[9] develops a flexible GISbased methodology for evaluating the potential locations of terminal Park and Ride facilities along urban passenger transport corridor. These studies, however, rarely consider the sum of construction costs and travel costs, and also lack the consideration of the abilities of Park and Ride facilities. Therefore, on the basis of traffic demand, this paper comprehensively takes the total travel costs of Park and Ride sites' construction and all traffic demand into account, in order to study the layout optimization of Park and Ride.

\section{II.GENERAL PRINCIPLES OF P\&R LAYOUT OPTIMIZATION}

The choice of Park and Ride location should follow certain principles which can initially determine the optional Park and Ride sites and make subsequent layout optimization simplified to some extent.

The general principles mainly include :(1)Park and Ride sites should be located in urban passenger traffic transport channels which have larger traffic volume, in order to effectively link with the large-capacity public transport system;(2)Park and Ride sites should be located in the places where generate the most car trips and have the biggest attraction to car trips within the service radius;(3)Park and Ride sites must be able to provide maximum comfort level for their facility users, such as setting in the terminus of bus lines, rail transport or nearby. As far as Beijing concerned, besides the general principles mentioned above to follow, there is another rule that is "don't set Park and Ride sites within the Fourth Ring". 


\section{III.BASIC MODELS OF P\&R LAYOUT}

In the planning of transportation hubs, the study of relevant models has been very mature. The traditional methods include the single variable orthocenter method in the layout of transportation hubs, the single variable differential method in the layout of transportation hubs, and cost analysis method. There are some classic models in the layout and location of transportation hubs, such as Covering model, median distance model in the plane, specific location model, etc ${ }^{[10]}$.

\section{A. Cost Analysis Method}

Cost analysis method takes the minimum costs in the whole system as the optimized target, and makes some simple financial calculations and chooses the sites whose total transportation costs are the lowest as the best layout of transportation hubs by comparison under the premise of given locations of the candidate hubs $\left(P_{1}, P_{2}, \cdots, P_{m}\right)$. The total cost of each candidate hub is calculated as follows:

$$
C_{i}=R_{i}+\sum_{j=1}^{n} d_{i j} W_{j} F
$$

Among: $R_{i}$ - The construction cost of candidate hub $i$;

$W_{j}$ - The amount of transportation demand from traffic generating source $j$;

$d_{i j}$ - The distance between traffic generating source $j$ and candidate hub $i$;

$F$ - The same unit transportation costs.

\section{B. Covering Model}

It is mainly introduced the covering model of the covering problems whose optimized objective is using the smallest number of location sites to cover all the requirements, that is using the least amount of transportation hubs for covering all the demand points.

$$
\min \sum_{j \in J} X_{j}
$$

s. $\mathrm{t}$

$$
\begin{aligned}
& \sum_{j \in N_{i}} X_{j} \geq 1, \forall i \in I \quad(2-3) \\
& X_{j} \in\{0,1\}, \forall j \in J \quad(2-4)
\end{aligned}
$$

Among: $I$-The set of all the transport demand points;

$J$-A collection of candidate hubs;

$d_{i j}$-The distance between demand point $i$ to candidate hub $j$;

$$
N_{i}=\left\{j \mid d_{i j} \leq D_{c}\right\}-\mathrm{A} \text { collection of all candidate }
$$

hubs that can cover demand point $i, D_{c}$ is the coverage distance;
$X_{j}$-Decision variables, if candidate hub $j$ is finally selected, $X_{j}=1$, otherwise $X_{j}=0$.

There are some unavoidable shortcomings when the two models mentioned above are directly applied to the layout optimization of Park and Ride. For example, the two models are all lacking the consideration of O-D demand that don't correspond to reality; they also lack the analysis of the abilities and capacities of Park and Ride sites; and there is no reference to assign a value to the covering distance $D_{c}$ in model 2.2 at present.

\section{IV.MODEL ESTABLISHMENT OF P\&R LAYOUT OPTIMIZATION}

\section{A.Ideas of Model Establishment}

First of all, according to the general principles of Park and Ride layout or any other layout optimization model, identify some candidate sites to get a collection of several initial points which may be selected by qualitative or quantitative methods.

Then divide the urban area of a city into two parts by some boundaries which are the virtual cables between any of the two initial transfer candidate sites. Within the boundaries, there are approximate to the centers of the city area which are considered as attractive areas of the transportation demand; the outside parts of the boundaries are approximate to the suburban areas of the city which are treated as the generating areas of the transportation demand. The fixed attractive points of transportation demand can be determined in the assumption of attractive areas, such as the city's commercial centers; then the fixed generating points of transportation demand can be determined in the generating areas as well, such as satellite towns around the city. Based on the division of transportation demand in the urban area, the structure of Park and Ride is shown in Fig.I. The traffic demand generated by traffic generating point $\mathrm{O}$ goes through the Park and Ride site $\mathrm{H}$, and finally reaches the traffic attractive pint $\mathrm{D}$.

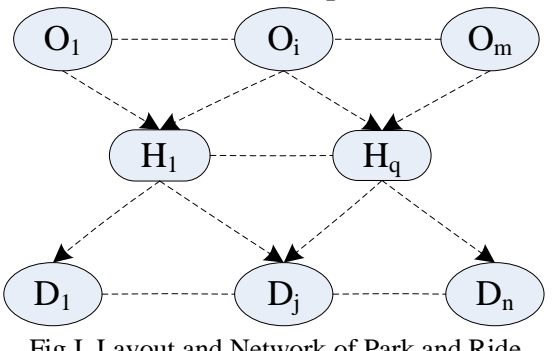

\section{B.Model Establishment}

The model assumptions include: (1)The quantity of traffic volume from traffic generating points $\mathrm{O}$ to traffic attractive points D that use Park and Ride program is known; (2)The traffic volume of traffic generating points can reach traffic attractive points by any one of the Park and Ride sites; (3)All traffic generating points that has transportation demand are within the attractive scope of all initial candidate Park and Ride sites; (4) The traffic volume of traffic generating points, the 
construction costs of alternative sites, one parking costs in each Park and Ride site, and the rate matrix of $O_{i} \rightarrow H_{k}$ and $H_{k} \rightarrow D_{j}$ are totally known and fixed.

Located in a city transportation system, there are $\mathrm{m}$ traffic generating points $O_{i}(i=1,2, \cdots, m), \mathrm{n}$ traffic attractive points $D_{j}(j=1,2, \cdots, n)$, and q candidate Park and Ride sites $H_{k}(k=1,2, \cdots, q)$ after preliminary selection. Here we design a layout optimization model which takes the total costs of $\mathrm{P} \& \mathrm{R}$ construction and personal travel demand as the minimum optimized goal and is supplemented by related constraints as follows.

$$
\begin{aligned}
\min W= & \sum_{i=1}^{m} \sum_{k=1}^{q} C_{i k} O_{i k}+\sum_{k=1}^{q} \sum_{j=1}^{n} C_{k j} D_{k j}+ \\
& \sum_{k=1}^{q}\left(C_{k} \sum_{i=1}^{m} O_{i k}\right)+\sum_{k=1}^{q} F_{k} X_{k} \\
= & \sum_{i=1}^{m} \sum_{k=1}^{q} C_{i k} O_{i k}+\sum_{k=1}^{q} \sum_{j=1}^{n} C_{k j} D_{k j}+ \\
& \sum_{k=1}^{q}\left(C_{k} \sum_{i=1}^{m} O_{i k}+F_{k} X_{k}\right)
\end{aligned}
$$

s. $\mathrm{t}$

$$
\begin{gathered}
\sum_{k=1}^{q} T_{i j}^{k}=T_{i j}, i=1,2, \cdots, m ; j=1,2, \cdots, n \\
O_{i k}=\sum_{j=1}^{n} T_{i j}^{k}, i=1,2, \cdots, m ; k=1,2, \cdots q \\
D_{k j}=\sum_{i=1}^{m} T_{i j}^{k}, k=1,2, \cdots q ; j=1,2, \cdots n
\end{gathered}
$$

$$
\begin{gathered}
\sum_{k=1}^{q} O_{i k}=A_{i}, i=1,2, \cdots, m(3-5) \\
\sum_{i=1}^{m} O_{i k}=\sum_{j=1}^{n} D_{k j}, k=1,2, \cdots, q \\
\sum_{i=1}^{m} O_{i k} \leq P_{k}, k=1,2, \cdots, q \\
\sum_{i=1}^{m} O_{i k}-M X_{k} \leq 0, k=1,2, \cdots q \\
O_{i k}, D_{k j} \geq 0, i=1,2, \cdots m ; \\
k=1,2, \cdots q ; j=1,2, \cdots n
\end{gathered}
$$

Among:

$W$ - Total costs produced by Park and Ride system;

$O_{i k}$-Traffic volume from traffic generating point $i$ to candidate Park and Ride site $k$;
$D_{k j}$ - Traffic volume from candidate Park and Ride site $k$ to traffic attractive point $j$;

$C_{i k}$-Unit rate from traffic generating point $i$ to candidate Park and Ride site $k$;

$C_{k j}$ - Unit rate from candidate Park and Ride site $k$ to traffic attractive point $j$;

$C_{k}$ - One parking costs of transfer users in candidate Park and Ride site $k$;

$F_{k}$ - Construction costs of candidate Park and Ride site $k$;

$T_{i j}^{k}$-OD traffic volume from traffic generating point $i$ to traffic attractive point $j$ by transferring at candidate Park and Ride site $k$;

$T_{i j}$ - OD traffic volume from traffic generating point $i$ to traffic attractive point $j$;

$A_{i}$ - Traffic volume generated from traffic generating point $i$;

$P_{k}$ - Traffic capacity of candidate Park and Ride site $k$;

$X_{k}$-Decision variables, if candidate Park and Ride site $k$ is finally selected, $X_{k}=1$, otherwise $X_{k}=0$;

$M-$ A positive infinity.

The objective 3-1 is to make the total costs of Park and Ride minimum in the whole urban transportation system; Constraint 3-2 restricts that the sum of traffic volume from traffic generating point $i$ through all candidate Park and Ride sites to traffic attractive point $j$ is equal to the OD traffic volume between traffic generating point $i$ and traffic attractive point $j$; Constraint 3-3 limits that the traffic volume from traffic generating point $i$ to candidate Park and Point site $k$ is equal to the total transfer traffic volume from traffic generating point $i$ to all traffic attractive points which go through candidate Park and Ride site $k$; Constraint 3-4 restricts that the traffic volume from candidate Park and Ride site $k$ to traffic attractive point $j$ is equal to the total transfer traffic volume from all traffic generating points to traffic attractive point $j$ which go through candidate Park and Ride site $k$; Constraint 3-5 restricts that the traffic volume from traffic generating point $i$ to all candidate Park and Point sites is equal to the traffic generating amount of traffic generating point $i$; Constraint 3-6 restricts that the traffic volume from all traffic generating points to candidate Park and Point site $k$ is equal to the traffic volume from candidate Park and Ride site $k$ to all traffic attractive points; Constraint 3-7 limits that the total traffic volume from all traffic generating points to candidate Park and Ride site $k$ must be less than its capacity; Constraint 
3-8 shows the corresponding relationship between candidate Park and Ride sites and its traffic volume, if the traffic volume from all traffic generating points to candidate Park and Ride site $k$ is not zero, $X_{k}=1$, which means candidate Park and Ride site $k$ is finally selected; Constraint 3-9 is a non-negative limit of traffic volume which is comprehensively measured by the rate matrix $C_{i k}$ and $C_{k j}$ from generating points through Park and Ride sites to attractive points, travel time, fuel costs, travel comfort and other aspects.

\section{V.NUMERICAL EXAMPLE}

In a transportation system, assume that there are 6 traffic generating points, 5 traffic attractive points, and 7 initial candidate Park and Ride sites that are determined by some general principles of $P \& R$ layout optimization. The position relationship of traffic generating points, traffic attractive points and candidate Park and Ride sites is shown in Fig.II.

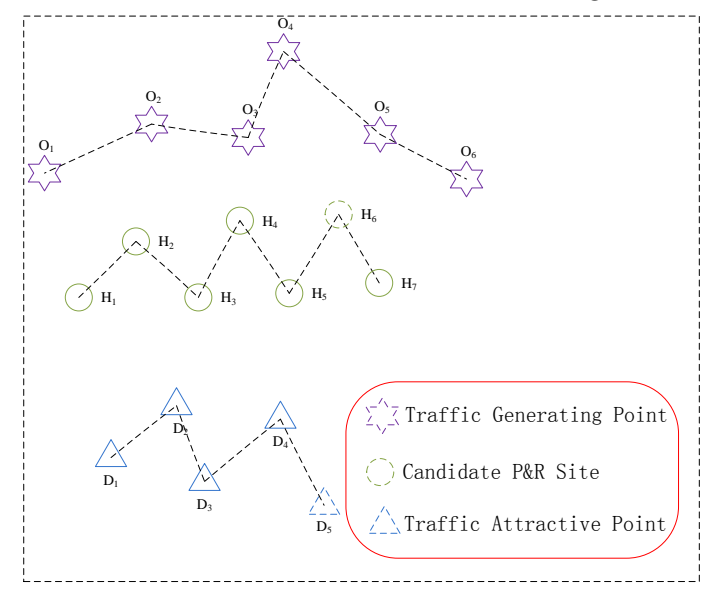

Fig.II The Example Diagram

Then we assume that the daily O-D traffic volume matrix between each traffic generating point to each traffic attractive point is shown in Tab.I, rate matrix of $C_{i k}$ which is consisted of the unit rate from traffic generating point $i$ to candidate Park and Ride site $k$ is shown in Tab.II, rate matrix of $C_{k j}$ which is consisted of the unit rate from candidate Park and Ride site $k$ to traffic attractive point $j$ is shown in Tab.III as follows.

Tab.I OD Table

\begin{tabular}{ccccccc}
\hline D & 1 & 2 & 3 & 4 & 5 & $\begin{array}{c}\text { Generating } \\
\text { Volume }\end{array}$ \\
\hline 1 & 300 & 190 & 150 & 90 & 60 & 790 \\
2 & 260 & 410 & 180 & 160 & 140 & 1150 \\
3 & 150 & 320 & 290 & 180 & 70 & 1010 \\
4 & 50 & 350 & 410 & 280 & 60 & 1150 \\
5 & 80 & 130 & 300 & 480 & 280 & 1270 \\
6 & 80 & 140 & 160 & 210 & 360 & 950 \\
Attractive & 920 & 1540 & 1490 & 1400 & 970 & 6320 \\
Volume & & & & & & \\
\hline
\end{tabular}

Tab.II. Rate Matrix of $C_{i k}$ (yuan)

\begin{tabular}{cccccccc}
\hline $\mathrm{H}$ & 1 & 2 & 3 & 4 & 5 & 6 & 7 \\
\hline $\mathrm{O}$ & 20 & 25 & 35 & 45 & 60 & 70 & 85 \\
2 & 30 & 20 & 30 & 40 & 50 & 60 & 70 \\
3 & 50 & 40 & 30 & 20 & 45 & 50 & 60 \\
4 & 90 & 75 & 60 & 45 & 30 & 40 & 60 \\
5 & 95 & 80 & 65 & 50 & 35 & 20 & 40 \\
6 & 100 & 85 & 70 & 50 & 40 & 30 & 20 \\
\hline
\end{tabular}

Tab.III Rate Matrix of $C_{k j}$ (yuan)

\begin{tabular}{cccccc}
\hline $\mathrm{D}$ & 1 & 2 & 3 & 4 & 5 \\
\hline 1 & 20 & 30 & 45 & 60 & 70 \\
2 & 25 & 20 & 35 & 55 & 65 \\
3 & 20 & 20 & 40 & 50 & 70 \\
4 & 50 & 35 & 20 & 40 & 60 \\
5 & 75 & 55 & 40 & 25 & 35 \\
6 & 75 & 60 & 45 & 30 & 55 \\
7 & 80 & 60 & 45 & 35 & 20 \\
\hline
\end{tabular}

And daily expenses in construction that are evenly allocated from the total construction costs of each candidate Park and Ride site and the parking fee for once are listed in Tab.IV, the accommodating capacity of each candidate Park and Ride site is shown in Tab. V as wll.

Tab.IV Costs of Transfer Sites (yuan)

\begin{tabular}{cccccccc}
\hline & $\mathrm{H}_{1}$ & $\mathrm{H}_{2}$ & $\mathrm{H}_{3}$ & $\mathrm{H}_{4}$ & $\mathrm{H}_{5}$ & $\mathrm{H}_{6}$ & $\mathrm{H}_{7}$ \\
\hline Construction Costs & 40000 & 35000 & 30000 & 38000 & 40000 & 35000 & 30000 \\
Transfer Costs & 15 & 12 & 10 & 14 & 15 & 12 & 10 \\
\hline
\end{tabular}

Tab.V Accommodating Capacity of Transfer Points

\begin{tabular}{|c|c|c|c|c|c|c|c|}
\hline & $\mathrm{H}_{1}$ & $\mathrm{H}_{2}$ & $\mathrm{H}_{3}$ & $\mathrm{H}_{4}$ & $\mathrm{H}_{5}$ & $\mathrm{H}_{6}$ & $\mathrm{H}_{7}$ \\
\hline $\begin{array}{c}\text { Accommodating } \\
\text { Capacity }\end{array}$ & 2000 & 2000 & 1500 & 1500 & 2000 & 1200 & 1400 \\
\hline
\end{tabular}

Based on the data assumed above, the model is solved by using Lingo mathematical software programming, finally get the optimized layout result under the premise of given initial candidate Park and Ride sites. As a result, select 4 Park and Ride sites that are $\mathrm{H} 2, \mathrm{H} 3, \mathrm{H} 5$ and $\mathrm{H} 7$ out of the 7 candidate sites as the final layout of Park and Ride. Each of them burdens the traffic transfer volume of 1940, 1450, 1730 and 1200 in sequence, and the total cost of the system is 600480.0 yuan.

\section{VI.CONCLUSIONS}

Based on Transportation Demand Management program, this paper analyzes the layout optimization of Park and Ride, and builds a mathematical model to optimize urban Park and Ride transfer system which comprehensively considers traffic volume, construction costs of Park and Ride and travel costs of transfer facilities users, in order to get the minimum total costs of the whole system. In the end, this paper also designed a case about it with solving the model by Lingo mathematics software so as to check the effectiveness of the model. As a result, this model has a certain reference value in the practical layout optimization of Park and Ride. In actual planning, preliminary layout can be determined according to some general principles, then use layout optimization model mentioned above for the further optimization, and finally get a reasonable and optimized Park and Ride layout project. The contents of this study also make much positive sense in further strengthening Transport Demand Management in urban traffic system. 


\section{ACKNOWLEDGMENT}

Here, Li Yuhong thanks her graduate tutor who is the second author-Professor He Shiwei. Both of Li Yuhong and He Shiwei thank National Basic Research Program of China(No.2012CB725403).

\section{REFERENCES}

[1] Liu Yan, Qin Huan-mei, Pan Xiao-song. Investigation and Analysis of Beijing Park \& Ride Demand[J]. Journal of Transportation Engineering and Information, 2011, 9(3):118124.

[2] Duan Zuo-hong. Existing Problems and Countermeasures Research on Park \& Ride in Beijng[J]. Road Traffic \& Safety, 2010(4):4-8.

[3] BERMAN O, HODGSON MJ, KRASS D. Flow Interception problems[M]. Berlin, Springer, 1995:389-426.

[4] MARK W, HORNER, SARA GROVES. Network Flow-based Strategies for Identifying Rail Park-and-Ride Facility Locations[J]. Socio-Economic Planning Science, 2007(41):255268.
[5] WANG JY, YANG TH, LINDESY R. Locating and Pricing Park-and-Ride Facilities in a Linear City with Deterministic Mode Choice[J]. Transportation Research Part B, 2004(38):709731.

[6] XIE Xiao-qian, XU Shi-kai. Model Study of Park-and-Ride Location[J]. Journal of Dalian Jiaotong University, 2012, 33(1):17-19.

[7] Fang Qing, Pan Xiaodong, Wu Zhong. Study on the model of location of urban park and ride lots based on genetic algorithm[J]. Sciencepaper Online, 2010, 5(10):763-766.

[8] Huang Yifeng, Qi Wenzhou. Metro Planning based on Particle Swarm Optimization Theory[J]. Urban Mass Transit, 2009(3):17-21.

[9] Liu Youjun, Yan Kefei. A GIS-Based Study on the Optim um Location of Urban Park \& Ride Facilities[J]. Transportation Technology \& Science, 2003(4):85-87.

[10] He Shiwei. Theory and Methods of Comprehensive Traffic Hub Plan[M]. Beijing, China Communications Press, 2012:86-87. 